\title{
Intussusception and spontaneous amputation of the esophagus
}

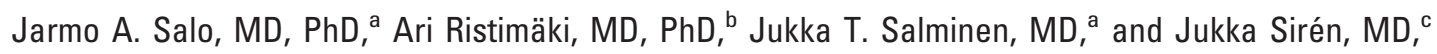
Helsinki, Finland

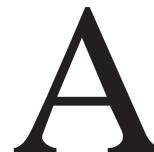

52-year-old woman with a history of hypertonia and hypothyroidism was hospitalized because of acute lymphatic leukemia. Earlier, she had had upper abdominal symptoms and had been given a diagnosis of a hiatal hernia and a duodenal ulcer.

She received chemotherapy (cytarabine-etoposide-mitoxantrone), leading to remission. However, neutropenia and severe bronchopneumonia subsequently developed. Bronchoscopy was performed for bacteriologic and fungal analysis. After bronchoscopy, she vomited and later reported having dysphagia. A foreign

From the Departments of Cardiothoracic Surgery (Section of General Thoracic and Esophageal Surgery), ${ }^{\mathrm{a}}$ Pathology, ${ }^{\mathrm{b}}$ and Gastroenterological and General Surgery, ${ }^{\mathrm{c}}$ Helsinki University Hospital, PO Box 340 (Haartmaninkatu 4), FIN-00029 HUS, Helsinki, Finland.

Received for publication Dec 20, 2001; accepted for publication Jan 11, 2002.

Address for reprints: Jarmo Salo, MD, Department of Cardiothoracic Surgery, Helsinki University Hospital, PO Box 340 (Haartmaninkatu 4), FIN00029 HUS, Helsinki, Finland (E-mail: jarmo.salo@ hus.fi).

J Thorac Cardiovasc Surg 2002;124:205-6

Copyright $(92002$ by The American Association for Thoracic Surgery $0022-5223 / 2002 \$ 35.00+0 \quad \mathbf{1 2 / 5 4 / 1 2 2 8 1 7}$

doi:10.1067/mtc.2002.122817

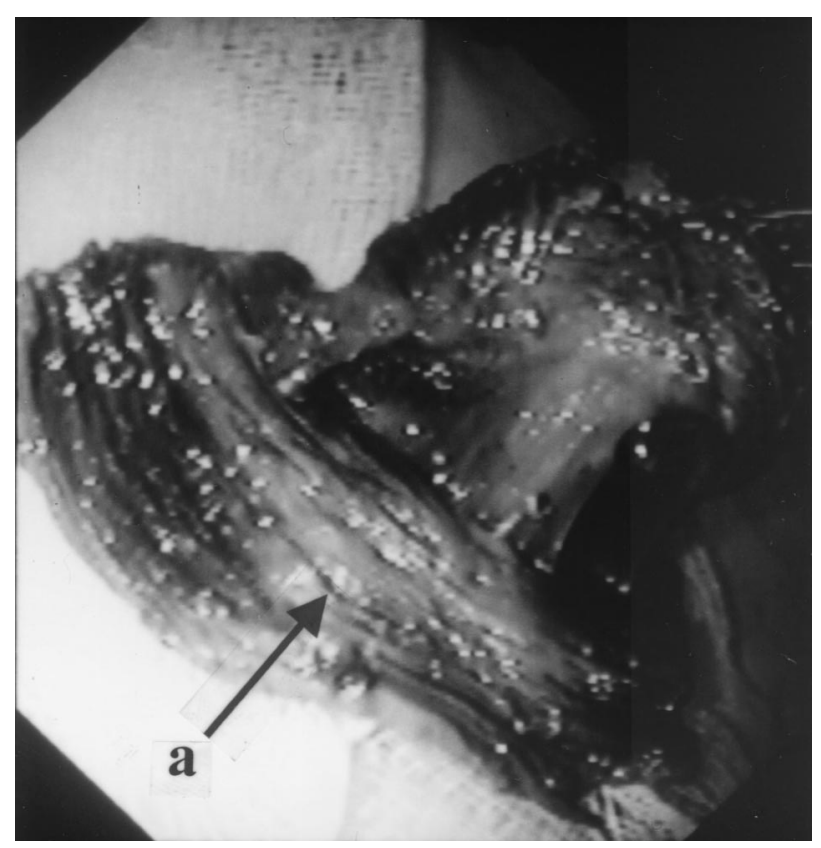

Figure 1. Intussuscepted segment of the esophagus showing the inner circular muscle layer (a) located superficially. body obstructing the lower esophagus was detected at endoscopy. The foreign body was pulled out and, after removal, a circular, slightly hemorrhagic, 4-cm-long, superficial mucosal ulceration was seen in the lower esophagus, but no signs of perforation were seen. The endoscope could easily be placed into the stomach and duodenum, both of which appeared to be normal. The foreign body was found to be a 4-cm-long segment of the lower esophagus, which, on more thorough inspection, was found to contain all the esophageal layers. As a sign of intussusception, the circular inner muscular layer was located on the outer surface of the removed specimen (Figure 1). In addition, histopathologic examination

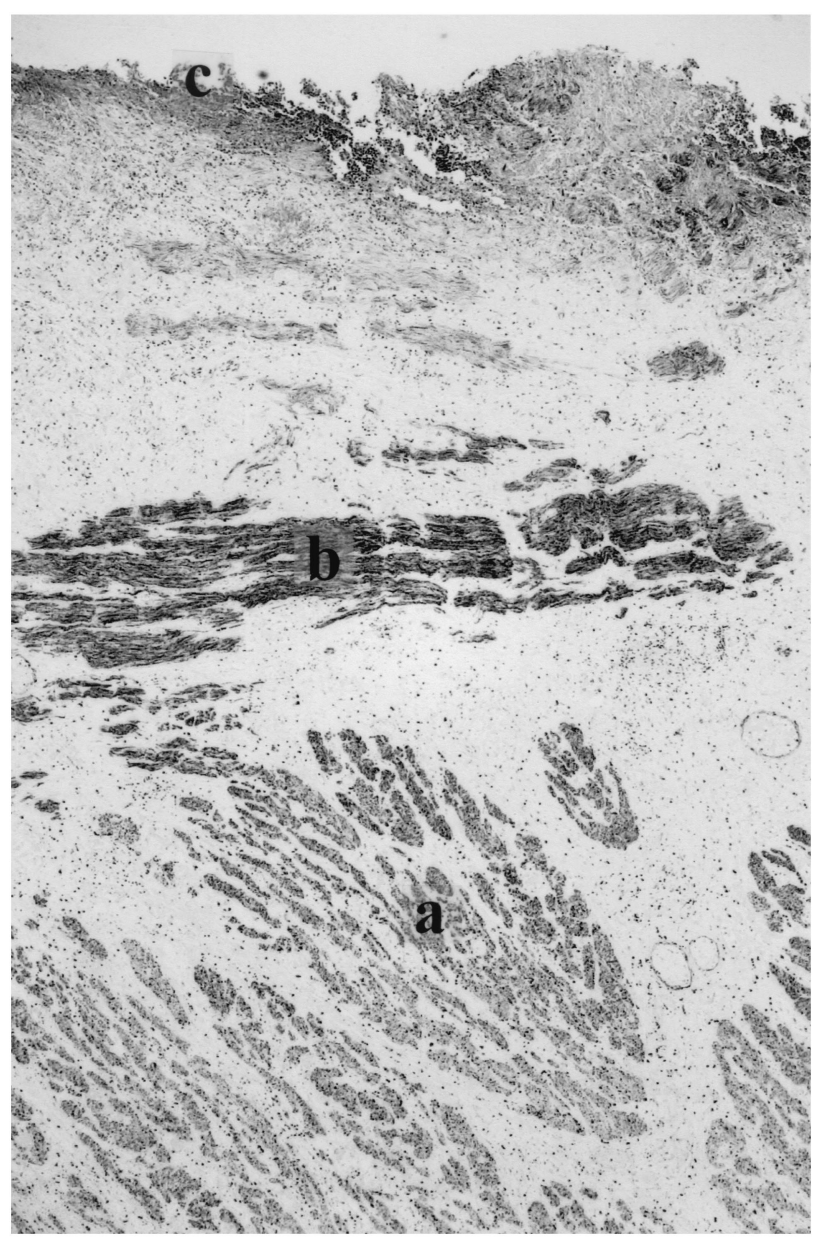

Figure 2. Histology of the specimen showing all layers of the esophageal wall: (a), circular muscle layer; (b), longitudinal muscle layer; and (c), largely necrotic mucosa. A monoclonal antihuman smooth muscle actin antibody was used for identification of the muscle cells. 
showed a segment of the esophagus containing both circular and longitudinal mucosal layers and a necrotic epithelium (Figure 2). After the procedure, the patient could eat and drink without difficulty during the following 4 weeks, although she reported some dysphagia. The esophageal wall healed without fistula. However, 4 weeks later, severe dysphagia developed. The circular stricture in the lower esophagus was palliated with dilation and a self-expandable metallic stent at endoscopy, and dysphagia once again resolved. A more aggressive approach to the stricture was prevented by a relapse of leukemia.

\section{Discussion}

In the English literature we could find no reports of spontaneous esophageal amputation followed by reparation without interruption. The cause of this incident is probably esophageal intussusception, which is an unusual complication of a hiatal hernia. The first case was reported in 1903 by Enderlen. ${ }^{1}$ Incidence of complete intussusception involving all layers of the esophageal wall is unknown, but partial gastroesophageal intussusception with mucosa prolapse might have an incidence of $1.4 \%$ on the basis of radiographic examination. ${ }^{2}$ Increased intra-abdominal pressure caused by vomiting, for example, is an important pathogenetic mechanism. Intussusception might be asymptomatic but can cause dysphagia, bleeding, anemia, and chest pain in certain patients. ${ }^{2}$ Radiologically, it might simulate a tumor. ${ }^{2}$ It is usually a transient phenomenon, resolving spontaneously. In some cases complete relief of symptoms has been achieved with fundoplication. ${ }^{3,4}$ In our patient severe vomiting increased intra-abdominal pressure, causing the entire thickness of the distal esophageal wall to infold and incarcerate inside the esophagus. Ischemic necrosis of the esophageal segment resulted, later amputating spontaneously. Fortunately, the esophageal wall healed without perforation or fistula. In addition, infection of the esophageal wall with Aspergillus species (as detected by both histologic and immunohistochemical stainings) might have contributed to the pathogenesis.

\section{References}

1. Enderlen J. Invagination der vorteren Magenwand in den Ösophagus. Deutsche Z Chir. 1903;69:60-7.

2. Keren RM. Mucosa prolapse and gastroesophageal intussusception: In: Castell DO, editor. The esophagus. Boston: Little, Brown and Company; 1995. p. 747-55.

3. Myllärniemi H, Saario I. A new type of sliding hiatus hernia. Ann Surg. 1985;202:159-61.

4. Gowen GF, Stoldt HS, Rosato FE. Five factors identify patients with gastroesophageal intussusception. Arch Surg. 1999;134:1394-7. 